\title{
Lesões não cariosas: Revisão de literatura
}

\section{Non-carious lesions: Literature review}

Recebido: 29/05/2021 | Revisado: 06/06/2021 | Aceito: 07/06/2021 | Publicado: 10/06/2021

Camila Ferreira Pinheiro
Centro Universitário das Faculdades Metropolitanas Unidas, Brasil
E-mail: camilaferreirapinheiro@gmail.com
Miréia Pinto Ferreira Melo
Centro Universitário das Faculdades Metropolitanas Unidas, Brasil
E-mail: miih_mireia@ @otmail.com
Rafael Rodrigues da Silva
Clínica Unisorriso, Brasil
E-mail: drrafael.unisorriso@gmail.com
Irineu Gregnanin Pedron
Universidade Brasil, Brasil
E-mail: igpedron@alumni.usp.br
Caleb Shitsuka
Centro Universitário das Faculdades Metropolitanas Unidas, Brasil
E-mail: cashitsuka@gmail.com

\section{Resumo}

As lesões não cariosas são um conjunto de lesões que podem ocorrer e apresentam característica de perda da estrutura dental sem estar ligada ao fator cárie, elas ocorrem por uma associação de alguns fatores, o que caracteriza uma etiologia multifatorial. Dentre essas lesões temos a abfração, abrasão, atrição e erosão. O objetivo deste trabalho foi realizar uma revisão de literatura comentando sobre as principais características das lesões não cariosas. Na metodologia foram considerados 12 arquivos no período de 2002 a 2020, com base em dados inglês e português: Scielo, PubMED, Google Acadêmico. Concluímos que é de extrema importância que o cirurgião-dentista deva realizar uma boa anamnese e exame clínico criterioso, para que possa dar um diagnóstico precoce e. Para um tratamento adequado deve ser realizado um planejamento de acordo com a lesão e seu estágio de severidade. É importante remover os fatores causais das lesões, assim evitando a progressão e surgimento de novas lesões.

Palavras-chave: Erosão dentária; Lesões não cariosas; Desgaste dos dentes.

\begin{abstract}
Non-carious lesions are a set of lesions that can occur and present a characteristic of loss of tooth structure without being linked to the caries factor, they occur due to an association of some factors, which characterizes a multifactorial etiology. Among these lesions we have abfraction, abrasion, attrition and erosion. The aim of this study was to conduct a literature review commenting on the main characteristics of non-carious lesions. In the methodology, 12 files were considered in the period from 2002 to 2020, based on English and Portuguese data: Scielo, PubMED, Google Scholar. We conclude that it is extremely important that the dentist must perform a good anamnesis and a careful clinical examination, so that he can give an early diagnosis and. For an adequate treatment, planning must be carried out according to the lesion and its stage of severity. It is important to remove the causal factors of the injuries, thus preventing the progression and appearance of new lesions.
\end{abstract}

Keywords: Tooth erosion; Non carious lesions; Tooth wear.

\section{Introdução}

A saúde é caracterizada como bem-estar físico e mental do indivíduo, sendo assim qualquer alteração desse estado irá afetar sua saúde no geral. Nos dias atuais muitos fatores como estresse e alimentação desbalanceada acabam influenciando na qualidade de vida do indivíduo. Tendo isso em vista, se torna cada vez mais importante realizar as consultas preventivas ao cirurgião-dentista, para que possa prevenir futuros problemas de sua saúde bucal.

Com o avanço estudos e fácil informação, foi possível ir mais afundo nos estudos sobre os fatores que causam doenças/lesões bucais e observou-se que em muitas dessas alterações, temos uma combinação de fatores que geram esses 
problemas, foi assim com as lesões cariosas e temos outros exemplos visíveis como o bruxismo e as lesões não cariosas (LNC). Sabendo disso, os profissionais da área da saúde devem cada vez mais trabalhar em conjunto, de maneira interdisciplinar, promovendo uma melhor condição de saúde para os pacientes. (Silva et al., 2020 e Carvalho et al., 2020)

Existem alguns tipos de lesões não cariosas, sendo elas: abfração, abrasão, atrição e erosão. O dente pode sofrer perda de estrutura dentária decorrente de lesões cariosas e não cariosas. As lesões não cariosas podem gerar alguns problemas, como desordem funcional, alteração na estética e até mesmo hipersensibilidade dentinária. (Kina et al., 2015)

A etiologia das lesões não cariosas é multifatorial, elas podem ser classificadas em: Abfração, Abrasão, Erosão e Atrição. Elas surgem devido a ações mecânicas, químicas (intrínsecas e extrínsecas), excesso de forças oclusais em direções erradas, fatores emocionais. Cada lesão apresenta sua característica única, na abfração pode ser visto um formato de cunha em sua lesão, com ângulos bem vivos.

O objetivo deste trabalho é descrever os tipos de lesões não cariosas através de uma revisão de literatura, abordando sobre seus aspectos clínicos, tratamento e etiologia.

\section{Metodologia}

Trata-se de uma revisão de literatura que foi abordado o tema de lesões não cariosas, para realizar a busca foram utilizadas as seguintes bases de dados, nos idiomas em língua inglesa e portuguesa: Scielo, PubMED, Google Acadêmico publicados no período de 2002 a 2020. Os descritores utilizados para busca foram: Lesões não cariosas, lesões cervicais não cariosas, erosão, atrição, abfração e abrasão. Para esta revisão foram considerados 16 artigos selecionados mais relacionados ao tema falando sobre etiologia, tratamento e características gerais.

\section{Revisão da Literatura}

\section{Lesões cervicais não cariosas}

De acordo com Kina et al., 2015 às lesões cervicais se caracterizam pela perda gradual de tecido mineralizado na região cervical do dente, desde que esteja associado há alguns fatores sem envolvimento das bactérias, além disso, sua etiologia é multifatorial e pode se originar de ações mecânicas e corrosões químicas que atingem esmalte ou dentina. Essas lesões não possuem uma predileção correta, podem atingir jovens e idosos devido a sua etiologia ser multifatorial, geralmente se manifesta principalmente no terço cervical da superfície vestibular de todos os dentes, sendo encontrada em sua maior parte na face vestibular dos dentes posterior e inferior. Quanto ao seu tratamento, é dependente de alguns fatores, como a sua etiologia, se o paciente se sente desconfortável, se a perda de estrutura é maior que 1mm, risco de exposição pulpar e o comprometimento estético etc. Inicialmente devemos orientar os pacientes em relação à sua dieta, alguns hábitos nocivos e higiene oral do paciente, para assim darmos início ao tratamento mais invasivo. Os autores Kina et al., 2015, comentam que um dos melhores tratamentos é utilizar a restauração com resina composta, devido ao seu bom custo-benefício, propriedades físicas, adesão, estética e facilidade de manipulação. Vale ressaltar que para se obter um bom prognóstico deve-se respeitar um protocolo adequado de restauração com materiais de qualidade, respeitando todo seu passo a passo criteriosamente.

Segundo Amaral et al., 2012, qualquer pessoa que possua dentes naturais pode desenvolver sinais de desgaste dental, mas muitos pacientes desconhecem suas consequências até que se atinja uma fase avançada. A maior parte dos pesquisadores acredita que a prevalência do desgaste dentário está aumentando, devido uma maior conscientização dos clínicos e manutenção dos dentes naturais por conta de uma dieta ácida. (Amaral et al., 2012; Maltarollo et al., 2020). 


\section{Lesões não cariosas}

As lesões não cariosas são classificadas em abfração, abrasão, atrição e erosão (corrosão). A atrição é caracterizada pela perda de tecido dentário devido ao contato com outro dentre, assim causando um desgaste. Clinicamente esse desgaste se apresenta bem definido e brilhante, com perda de estruturas nas cúspides e nas faces incisais. Ele geralmente ocorre por hábitos parafuncionais como bruxismo ou contato entre os dentes, um dos fatores predisponentes que acaba fazendo com que ocorra a atrição é a questão de ter um dente antagonista feito de porcelana. A abrasão é uma lesão que ocorre uma perda de estrutura realizada por um processo mecânico ou uso de materiais abrasivos contra os próprios dentes, como por exemplo: escovação incorreta, dentifrício abrasivo, cerdas duras etc. Analisando clinicamente ela se apresenta com formato de "'V'" na região cervical. Esta lesão também pode ocorrer na face oclusal, porém com características diferentes da atrição, a abrasão oclusal envolve toda face oclusal e não temos hipersensibilidade. A ocorrência dela na face oclusal é resultante da dieta, mastigação de materiais abrasivos ou da exposição contínua à poeira e areia, outros fatores que podemos citar são segurar o cachimbo e o grampo de cabelo com os dentes. Um caso curioso é que comer sementes de girassol seca também pode causar essa lesão. A abfração é causada pelo estresse do dente decorrente de forças oclusais, acontece na região cervical por ser mais frágil à esse tipo de estresse. Clinicamente ela se apresenta com formato de "V' ou cunha com ângulos internos e externos bem visíveis, também pode se manifestar em formato de ' $C$ ', ou semicircular (região oclusal). Uma característica única da abfração é que a lesão pode se estender subgengivalmente. Podemos ter dois casos de paciente com erosão, onde cada um terá sua própria característica. Primeiro é quando a erosão progride de maneira lenta, geralmente é causado pela dieta (comidas e bebidas), é assintomática e o paciente não procurará tratamento, assim a lesão irá evoluir sem sintomas perceptíveis. Em contrapartida podemos ter uma erosão que progride rápido, nesses casos ela está relacionada à desordens alimentares, apresentando hipersensibilidade e alteração negativa na estética, geralmente os pacientes nesse tipo de caso procuram o tratamento. Para os casos de erosão dentária, consideramos a aplicação de flúor o padrão ouro. Existem estudos in vitro com outras substâncias (gel de fluoreto de fosfato acidulado modificado, biovidro, Caseína-fosfopeptídeo estabilizado nanocomplexos de fosfato de cálcio amorfo etc.) que mostraram ser benéficas, porém ainda não tem aplicação clínica para os materiais apesar de serem bem promissores, por isso são necessários mais estudos clínicos para confirmar a eficácia desses materiais, pois os estudos in vitro não confirmam sua eficácia em situações clínicas.

As LNC são lesões que observamos com certa frequência no consultório, elas acabam afetando a integridade estrutural do dente, podem gerar hipersensibilidade dental, aumentar o acúmulo de placa, causar perda de vitalidade pulpar e prejudicar a estética. Sobre a sua incidência, a literatura aponta que temos uma expectativa de aumentar os casos conforme os pacientes vão chegando em uma idade mais avançada sem perder elementos dentais, porém, existem alguns fatores que podem afetar nessa expectativa, como alguns procedimentos clínicos, hábitos e caso tenha um diagnóstico precoce dessas lesões.

Segundo Xavier et al, 2012, as lesões não cariosas não têm nenhuma associação estatisticamente comprovada entre o tipo de lesão, idade, gênero e dieta pois elas apresentam inúmeros tipos de fatores etiológicos dentre eles temos: alimentos ácidos, processo abrasivo, hábitos parafuncionais entre outros. Vale ressaltar que, caso esses processos apareçam simultaneamente, as incidências dessas lesões são aumentadas.

\footnotetext{
Abrasão

A abrasão é caracterizada como um processo de desmineralização da estrutura dentária ou restauração, onde não se tem placa bacteriana. Essa perda ocorre de maneira lenta, gradual e progressiva decorrente dos hábitos nocivos do paciente. A área mais atingida são as zonas cervicais, assim podendo gerar sensibilidade, exposição e/ou necrose pulpar. Uma das
} 
etiologias que está ligada à abrasão é a maneira inadequada de escovar os dentes, quando se coloca muita força, podendo estar associado ao uso de dentifrício abrasivo, acaba causando um desgaste mecânico.

A abrasão é caracterizada como um desgaste patológico do tecido duro por forças mecânicas, porém não estando relacionado à oclusão, esta perda de estrutura dental pode ser difusa ou localizada como pode ser vista na Figura 1. Os seus fatores etiológicos estão relacionados com a higienização de maneira incorreta (força, técnica, frequência). Um fator determinante para que ocorra lesão nas superfícies é o uso incorreto de fio dental ou utilizar palito de dente. Clinicamente se apresenta com superfície dura, altamente polida e contorno irregular, sendo os principais dentes afetados os caninos e prémolares. Seu tratamento preventivo mais comumente adotado é escolher um dentifrício menos abrasivo, além de uma correta escovação, caso o uso de palitos de dente deve aconselhar o paciente a parar com o uso. Em alguns casos pode surgir sensibilidade, para esse tipo de caso podemos usar agentes dessensibilizantes e restauração em resina composta.

Figura 1: Abrasão.

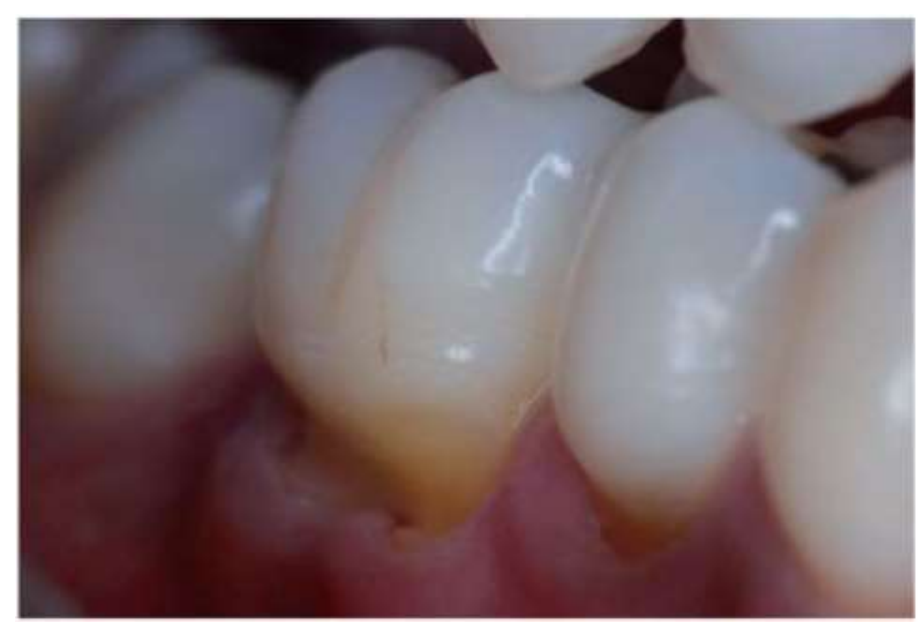

Fonte: (Amaral et al., 2012).

\section{Atrição}

A atrição é caracterizada com o desgaste fisiológico/patológico do dente ou restauração, que acaba sendo gerado pelo apertamento dos dentes voluntário ou involuntário. Este problema pode ocorrer nos 2 tipos de dentição, geralmente ele é mais presente nas superfícies oclusais, incisais ou linguais de dentes anteriores superiores, já em dentes inferiores atinge em sua maior parte na face vestibular, ilustrada na Figura 2. (Tuñas et al., 2016; Amaral et al., 2012; Beiriz et al., 2020; Warreth et al., 2019) 
Figura 2: Atrição.

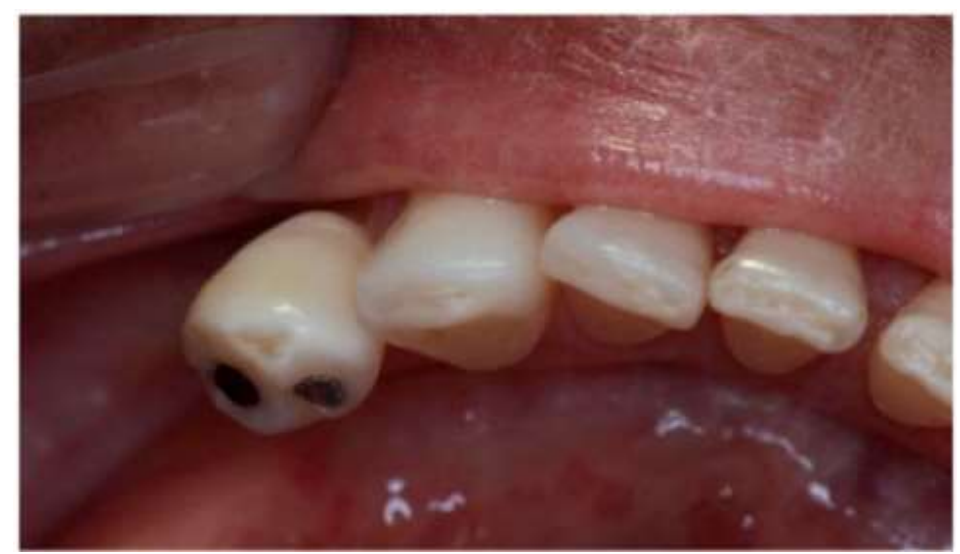

Fonte: (Amaral et al., 2012).

\section{Abfração}

A abfração é quando ocorre uma perda dentária na parte cervical do dente, acontece devido a forças oclusais aplicando uma tensão no fulcro cervical levando ao rompimento do esmalte. Uma característica exclusiva dessa lesão é o formato de cunha. Essa lesão tem maior incidência nos dentes inferiores devido ao seu diâmetro coronário da região cervical ser menor, comparado à arcada superior. A abfração pode ser caracterizada como uma perda patológica dos tecidos duros dentários, que provém de forças oclusais traumáticas, gerando flexões dentais causando a perda de esmalte, dentina e cemento. Sua etiologia como já foi comentada anteriormente é multifatorial, tendo como origem interferências oclusais, apertamento dos dentes ou esforços mastigatórios, essas características podem ser observadas na Figura 3. "De acordo com a teoria da flexão do dente, forças parafuncionais em áreas em que ocorrem interferências podem proporcionar a um ou mais dentes fortes esforços tensionais, compressivos ou de cisalhamento. Essas forças se concentram na junção cemento-esmalte, promovendo microfraturas nesse tecido, através das quais moléculas de saliva e água penetram, tornando a região suscetível ao efeito solubilizador de ácidos e efeito abrasivo da escovação. Acredita-se que, com o tempo, as microfraturas evoluem perpendicularmente ao longo eixo dos dentes sob pressão até o esmalte e a dentina serem "fraturados", culminando com defeitos em forma de cunha com bordas afiadas." (Pereira, 1995). Observando clinicamente a lesão de abfração é visto em formato de cunha, geralmente profundas e margens definidas, na maior parte dos casos atinge os dentes inferiores, geralmente afeta um único dente. Para seu tratamento devemos lembrar de realizar uma estabilização oclusal, caso haja presença de sensibilidade dental, podemos usar agentes dessensibilizantes, laser terapia ou até mesmo restauração em resina composta.

Existem vários índices propostos que avaliam/monitoram a progressão das lesões de abfração. O "The Smith and Knight" é uma forma, usa uma escala de 0 até 4 para medir a profundidade da lesão, sendo o número $0=$ não tem alteração de contorno, $1=$ possui uma mínima alteração no contorno, $2=$ Lesão com profundidade menor que 1 mm, $3=$ Lesão com profundidade entre 1 e $2 \mathrm{~mm}$ e 4= Lesão com profundidade maior que $2 \mathrm{~mm}$ ou apresentar exposição de dentina secundária ou polpa. Quando o paciente possui uma lesão que não compromete o dente em função ou vitalidade pulpar, uma estratégia interessante é realizar o acompanhamento desta lesão, levando em consideração a idade do paciente e as possíveis complicações que irão poder surgir, em casos mais graves que pode gerar o comprometimento do dente (exposição pulpar, fratura) devemos fazer uma intervenção, em casos que vemos uma profundidade menor que 1 mm temos uma oportunidade de realizar o acompanhamento, que deve ser feito a cada 6 meses ou de 12 em 12 meses. Outra alternativa interessante em casos de retração gengival, podemos realizar um procedimento cirúrgico que é o recobrimento de raiz, é feito levando em conta a classificação de Miller, de acordo com essa classificação, quando temos retração gengival classe I e II e não observamos perda 
de inserção periodontal óssea e interdental podemos esperar recobrimento completo da raiz. Nos casos de classe III e IV de retração gengival e uma perda moderada ou severa de tecido ósseo interdental, podemos esperar o recobrimento parcial ou insucesso deste procedimento. Além disso, também é importante observar caso o dente apresente rotação ou extrusão. Se combinar o procedimento restaurador aliado a esse procedimento cirúrgico, irá resultar em um prognóstico melhor, devido à melhor visualização do procedimento. (Nascimento et al., 2016)

Figura 3: Abfração.

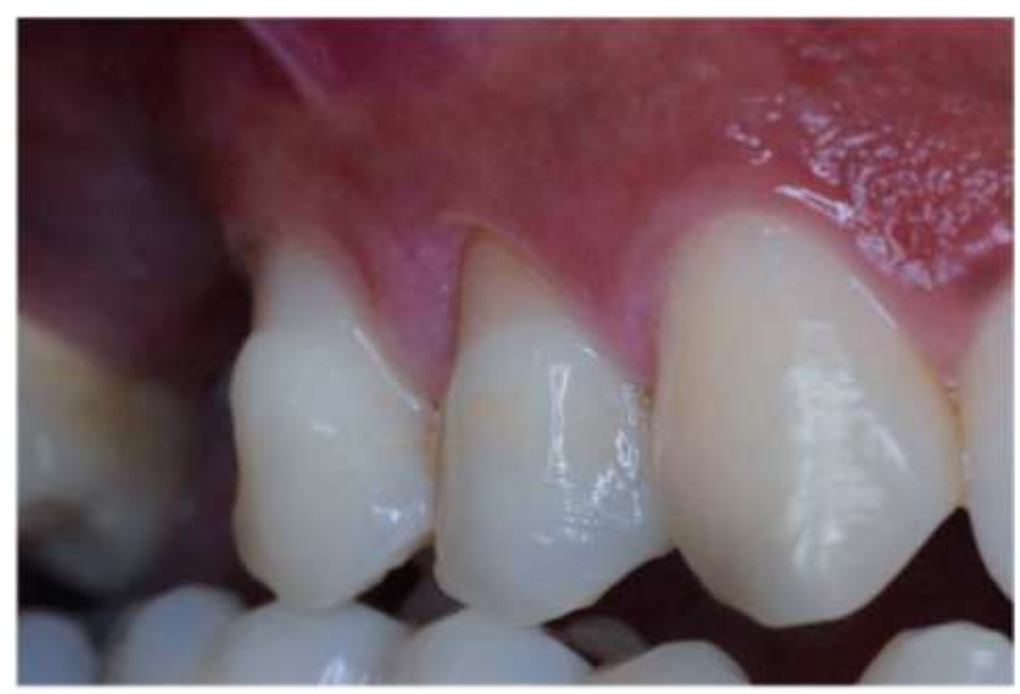

Fonte: (Amaral et al., 2012).

\section{Erosão}

A erosão dentária é caracterizada por perda de estrutura dentária proveniente de uma ação química, sem estar associada a alguma bactéria. Sua etiologia pode ser dividida em dois tipos, sendo extrínseco (suco de frutas, bebidas energéticas, refrigerante, alimentos ácidos em gerais e alguns medicamentos) e intrínseco (Refluxo, vômito frequente) (Maltarollo et al., 2020). O uso de dentifrícios pode influenciar o processo de lesões não cariosas, podemos ver que está associado, porém não é apenas esse fator que leva a evolução da doença não cariosa, pois se a pessoa tiver uma má escovação e usar um dentifrício que seja bastante abrasivo e uma dieta rica em alimentos com pH ácido. A Figura 4 apresenta a característica do desgaste erosivo nos dentes.

Figura 4: Erosão.

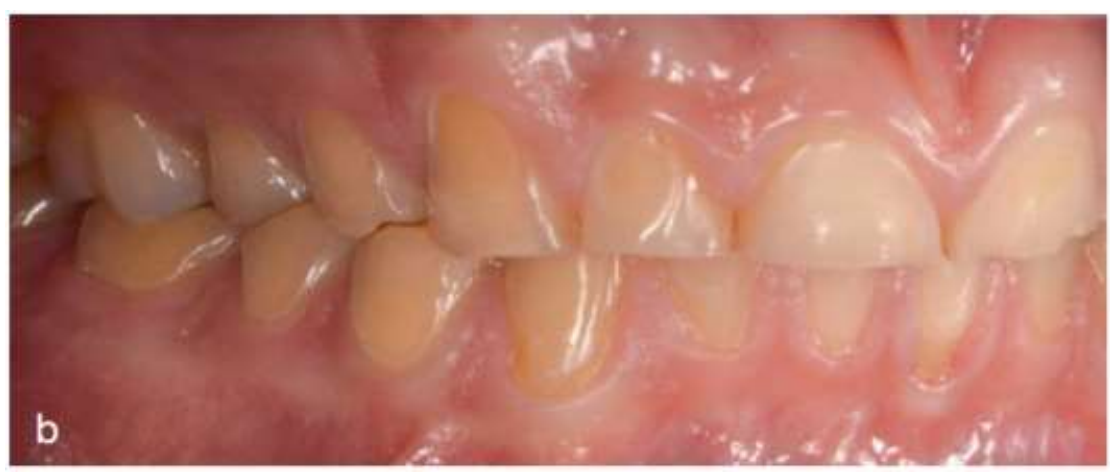

Fonte: (Warreth et al., 2019). 
A erosão é a perda irreversível de substância dentária decorrente de processos químicos, que não possuem o envolvimento de bactérias. Inicialmente ocorre uma desmineralização da camada mais superficial do esmalte que com o tempo pode chegar até a camada dentinária (Shitsuka, Tello \& Corrêa, 2016). Clinicamente podemos observar que o esmalte apresenta um aspecto sem brilho ou fosco e possuem degraus e cavidades. Em relação às cúspides, elas se encontram arredondadas e algumas cavidades podem alcançar até a dentina (Shitsuka et al., 2020). Sua etiologia pode estar relacionada à fatores intrínsecos ou extrínsecos, sendo multifatorial, assim para dar início ao tratamento devemos remover os fatores causais, para que seja elaborado um procedimento restaurador em resina composta, cimento de ionômero de vidro etc., para que assim tenha um controle da sensibilidade, melhora na função e estética. Para que o tratamento tenha sucesso e longevidade, é necessário orientar o paciente em relação à sua dieta, caso a sua etiologia tenha sido relacionada a fatores extrínsecos.

\section{Etiologia}

A etiologia das LNC é multifatorial e ocorre uma interação de diversos mecanismos entre si de maneira complexa e não temos o envolvimento de microrganismos nessas interações. Pode-se observar que fatores influenciadores de dois tipos: intrínsecos e extrínsecos. Os extrínsecos normalmente são relacionados à nossa dieta e medicação, sendo mais encontrados em uma dieta com alimentos que possuem $\mathrm{pH}$ de aproximadamente 4.5 como as frutas, refrigerantes e outras bebidas carbonatadas, por isso devemos levar em conta o pH crítico do esmalte e dentina, sendo respectivamente 5.5 e 6.5. Em relação aos fatores intrínsecos vemos que os principais causadores seriam o refluxo gastroesofágico e regurgitação, devido ao baixo pH do suco gástrico que acaba causando uma destruição severa do esmalte e dentina comparando se comparado com os fatores extrínsecos. Além disso podemos ter a associação a dois tipos de lesões, já que os após casos de vômitos geralmente é feito uma higiene bucal vigorosa, assim gerando uma perda da estrutura dentária por abrasão. Além dos fatores comentados temos alguns processos mecânicos que provém de hábitos nocivos (roer unha, palito de dente, morder lápis, ranger dentes), assim gerando uma nova lesão não cariosa. Alguns estudos mostraram que a abfração dentre as lesões não cariosas é a mais presente no sexo feminino, porém outros estudos mostram que a prevalência de lesões cervicais acaba crescendo com a idade e afeta mais o sexo masculino. O seu diagnóstico normalmente é feito na anamnese, através do exame visual e tátil, caso o dentista não identifique esse problema, essas lesões podem gerar um aumento de retenção de placa, assim causando outros problemas, com isso vemos a importância de identificar essas lesões em estágio inicial e planejar um tratamento adequado para cada caso. (Beiriz et al., 2020)

Oliveira et al., 2020, mostra que essas lesões não têm predileção por sexo ou idade, uma característica que pode se ver é que com o envelhecimento vemos um efeito acumulativo dessas lesões (progressão). Quanto a sua etiologia ela é multifatorial, pois dificilmente veremos uma lesão associada apenas em um fator etiológico. O local que essas lesões mais atingem é na região cervical, por conta das suas características estruturais e morfológicas, já que a camada de esmalte nessa região é mais fina, contém uma concentração maior de apatita carbonatada a direção dos prismas é vertical e linear, também tem o fato da ligação entre esmalte e dentina dessa região ser menos resistente. $\mathrm{O}$ autor mostra que podemos dividir os fatores etiológicos em 2 tipos, sendo mecânico e químico. Dentro dos fatores mecânicos podemos citar alguns grupos de risco como: portadores de bruxismo, desoclusão de dente posterior, paciente com tratamento ortodôntico. Como exemplos de fatores mecânico temos: escovação inadequada, cerdas duras nas escovas, dentifrício abrasivo, uso incorreto de escova interdental, além desses fatores vimos que algumas profissões têm uma maior prevalência devido ao exercício de suas funções é o caso de: músicos, alfaiates e carpinteiros. A erosão está ligada a fatores químicos, que podem ser divididos em intrínsecos e extrínsecos.

Os fatores extrínsecos estão associados aos hábitos alimentares, logo estando mais associado aos jovens por conta da introdução de um novo tipo de alimentação, já que nessa idade ocorre uma mudança no estilo de vida, utilizando com mais 
frequência os refrigerantes, isotônicos, sucos cítricos etc. Também vemos algumas profissões com maior índice de prevalência nesse tipo de lesão, como por exemplo: atletas profissionais, enólogos (degustador de vinho), profissionais de indústria (petrolífera, automobilística, metalúrgica etc.) No de atletas e enólogos é decorrente da ingestão recorrente de bebidas mais ácidas, já os profissionais de indústria são devido à condição do ambiente em que trabalham, já que são expostos rotineiramente a alguns tipos de ácidos. Para ocorrer a erosão através de fatores intrínsecos, o paciente normalmente apresenta alguns problemas como: bulimia nervosa, alteração no fluxo salivar (Diabetes Mellitus, Síndrome de Sjögren), refluxo gastroesofágico etc.

Segundo Pinheiro et al., 2020 a erosão uma lesão muito frequente e está associada ao estilo de vida dos pacientes. Á respeito da desmineralização no esmalte, ela é caracterizada clinicamente por apresentar formato de pires ou '’U', bem delimitado, com pouca profundidade, fosca, lisa e polida. Quando chegamos na dentina observamos uma manifestação bem rápida, devido ao seu composto ser menos mineralizado e menos resistente que o esmalte. Essa lesão inicialmente gera um problema estético, pois a camada de esmalte perdida revela um aspecto amarelado vindo da dentina. Podemos observar em casos mais graves outras complicações, como: diastemas, alteração da DVO, dor muscular e DTM. Seu diagnóstico é feito a longo prazo, devido ao processo de desmineralização do esmalte ser um pouco lento. Para seu tratamento é fundamental orientar o paciente sobre a mudança para um estilo de vida saudável, hábitos de higiene adequados. Caso haja necessidade encaminhar o paciente para o médico especialista em distúrbio gastroesofágico ou psicólogo caso o paciente tenha transtornos alimentares como a Bulimia. Além das suas orientações, o CD deve realizar um plano de tratamento adequado para cada caso, devido a sua etiologia ser multifatorial e diferente para cada pessoa, visando alívio da sintomatologia e o controle da evolução da doença.

As lesões não cariosas geralmente são consequência da ação sinérgica de dois ou três fatores etiológicos específicos da lesão. Sobre a sua prevalência temos valores que podem variar $5 \%$ até $85 \%$, essa variação está relacionada com a sua variedade da faixa etária e por incluir os dois gêneros em estudos populacionais. Um fator em comum que foi observado neste estudo é que a prevalência, severidade e progressão dessas lesões, aumentam com a idade. (Peumans et al., 2020)

\section{Tratamento}

O tratamento restaurador visa reestabelecer a estética e função. Clinicamente a abfração se apresenta em forma de cunha ou ' $V$ ', essas lesões podem ser mais profundas do que larga, apresentando ângulos internos e externos bem definidos. Normalmente ela está associada às lesões de abrasão e erosão. Seu diagnóstico é feito clinicamente observando as características da lesão e a oclusão do paciente (contato prematuro, apertamento, sobrecarga oclusal). Para obter sucesso no tratamento devemos reajustar a oclusão, algumas opções para remover o fator causal da lesão, podemos fazer isso através da confecção de placas miorrelaxantes, cirurgia ortognática e tratamento ortodôntico. Após concluir essa etapa podemos seguir para um plano de tratamento diferencial, em vista que cada caso tem suas peculiaridades, ao final de tudo realizamos a restauração do elemento. Em seu aspecto clínico, a abrasão é bem semelhante à abfração, porém a sua superfície é rasa e polida, além de estar associada à retração gengival, atingindo em maior parte no canino e pré-molar devido a sua convexidade. Seu diagnóstico é igual as outras lesões não cervicais (abfração, erosão). Para seu tratamento devemos iniciar com agentes dessensibilizantes, laserterapia etc., vale ressalvar que devemos orientar o paciente a respeito da sua higiene e indicar o dentifrício adequado. Um ponto em comum de todas as lesões cervicais não cariosas é o fato de poder ocorrer a sensibilidade dentária, uma maneira de resolvermos esse problema é fazer um tratamento aplicando agentes dessensibilizantes e laserterapia. 
Warreth et al., 2019 e Anjos et al., 2020, comentam que as lesões não cariosas são de condição multifatorial e possuem uma etiologia complexa, sendo assim devemos ter um diagnóstico preciso e o mais precoce possível para que seja feito um plano de tratamento adequado.

Uma das opções de tratamento para lesões cervicais não cariosas é realizar a restauração em resina composta. Shaalan et al., 2017, comparam a eficácia de resina composta convencional e resina composta fluida, conseguindo o seguinte resultado: Para as lesões cervicais não cariosas não apresentaram diferenças estatísticas ou clínicas entre os dois tipos de resina, porém as resinas fluidas têm uma maior capacidade de aliviar tensões nas interfaces adesivas, tem o efeito de quebra de tensão nas áreas de tensão térmica, oclusal e sofre menos contração de polimerização, devido a isso podemos utilizar sem nenhum malefício as resinas compostas fluidas.

\section{Discussão}

As lesões não cariosas são lesões que não estão associadas à doença cárie, apresenta uma etiologia multifatorial que pode estar ligada à alimentação, hábitos nocivos, medicamentos, profissão etc. Existem quatro tipos sendo: abrasão, abfração, atrição e erosão. Cada uma apresenta uma característica e etiologia específica, mas no geral costumam apresentar um manejo de tratamento parecido.

As LNC são mais prevalentes na população adulta, aumentando gradualmente a porcentagem conforme a idade avança. No geral essas lesões são mais presentes no sexo masculino, porém identificamos um artigo que mostrou um maior índice de prevalência em lesões de abfração para o sexo feminino. (Marinescu et al., 2017 e Beiriz et al., 2020)

Sobre a erosão a literatura atual mostra que o termo mais adequado para se utilizar é '’Biocorrosão", por englobar os processos químicos, bioquímicos e eletroquímicos de dissolução do tecido dental mineralizado. (Oliveira et al., 2020, Warreth et al., 2019 e Nascimento et al., 2016).

Um fato interessante que foi identificado em alguns estudos, é que existem profissões que podem estar associada ao desenvolvimento de LNC. Referente à biocorrosão extrínseca, pode estar associada à exposição que um indivíduo tem em seu ambiente de trabalho, temos exemplos de alguns profissionais que trabalham em indústria petrolíferas, automobilísticas, metalúrgicas, pirotécnicas e fertilizantes, já que estão frequentemente expostos ao ácido sulfúrico, hidroclorídrico, nítrico e crômico. Algumas outras profissões como o músico, alfaiate e carpinteiro estão associados ao maior desenvolvimento da abfração e atrição, por conta do exercício de suas funções acaba utilizando a boca como '’auxílio" para segurar algum objeto. (Oliveira et al., 2020)

Sobre o tratamento, não encontramos nenhum tipo de protocolo que sempre funcionará em $100 \%$ dos casos, porém a literatura sugere uma sequência estratégica focando em alguns pontos, gerando um tipo de "protocolo" que consiste nas seguintes etapas: prevenção, monitoramento, tratamento da hipersensibilidade dentinária, tratamento restaurador e cirurgia de recobrimento de raiz (pode estar associada ou não com tratamento restaurador). (Peumans et al., 2020, Pinheiro et al., 2020 e Kina et al., 2015).

O tratamento preventivo tem o intuito de não deixarmos que as lesões existentes não evoluam e que não ocorra o desenvolvimento de novas lesões, posteriormente iremos aumentar a longevidade das restaurações. O tipo de tratamento preventivo irá variar dependendo da etiologia da lesão. Vale ressaltar a importância de realizar um bom diagnóstico para que seja feito o protocolo de tratamento adequado. (Peumans et al., 2020 e Oliveira et al., 2020)

Realizar o monitoramento é um processo para decidir o plano de tratamento, avaliando a progressão da lesão e quando se deve intervir, também foi observado que com o monitoramento identificamos as atividades das lesões, evitando que a mesma chegue à um ponto crítico. (Peumans et al., 2020) 
O tratamento de hipersensibilidade dentinária tem o objetivo de amenizar a dor do paciente, em vista que os estágios iniciais das lesões acabam causando dor ao paciente. Podemos lançar mão de diversos agentes dessensibilizantes para combater essa hipersensibilidade. (Shitsuka et al., 2015; Peumans et al., 2020 e Pinheiro et al., 2020)

O intuito de realizar o tratamento restaurador é estabelecer a estética, função e impedir a progressão da lesão. Identificamos um artigo que comparou a aplicação de compósito resinoso fluido e convencional, no estudo feito foi exposto que ambas não possuem diferenciação estatística e clínica, porém tem a habilidade de aliviar estresse nas interfaces adesivas e alívio na tensão térmica e oclusal, assim sendo mais recomendada para lesões não cariosas. (Shaalan et al., 2017)

A indicação de tratamento para recobrimento de raiz nem sempre é necessária, mas podemos realizar a restauração em resina associada com ele. O seu objetivo é melhorar a parte estética em casos de recessão gengival. (Peumans et al., 2020)

. É importante que o ensino Odontológico seja de forma remota ou presencial, transmita essas orientações para que sejam aplicadas na prática profissional (Santo, 2016; Camacho et al., 2020; Fernandes et al., 2020; Rocha et al., 2020).

\section{Conclusões}

Atualmente as lesões não cariosas estão mais frequentes nos consultórios odontológicos, elas podem se tornar um desafio para o cirurgião-dentista, já que ela advém de uma etiologia multifatorial. Com isso em mente, é de extrema importância que o profissional deva buscar a maior quantidade de informações possíveis na anamnese e realizar um exame clínico criterioso, para que possa dar um diagnóstico preciso e o mais precoce possível. Vimos também que para realizar um bom tratamento devemos realizar um planejamento de acordo com a lesão e estágio que ela se encontra, sendo individual para cada caso. O ponto importante para iniciar o tratamento é remover os fatores causais da lesão, assim evitando sua progressão e surgimento de novas lesões. Diante do exposto é importante a realizaçãode visita periódica ao cirurgião-dentista, já que assim é possível identificar as lesões em seus estágios iniciais não deixando elas progredirem e alterando negativamente a estética, função ou gerar um problema como a hipersensibilidade dentinária.

\section{Referências}

Amaral S M, Abad E C, Maia K D, Weyne S, Oliveira M P R P B, \& Tunãs I T C. (2012). Lesões não cariosas: o desafio do diagnóstico multidisciplinar. Arquivos Int. Otorrinolaringol. [Internet]. Mar; 16( 1 ): 96-102.

Anjos R S, Cabral L F S, Menezes V A, Colares V, Franca C, \& Godoy F. (2020). Bruxism associated with mental factors in adolescentes: na integrative review. RSD [Internet]. 9(9). e737997590.

Beiriz R K A, Silva J S, Dantas R P, Silva I B L, Ramos A T P R, \& Cabral L L. (2020). Fatores associados as lesões cervicais não cariosas nos dias atuais. Ciências Biológicas e de Saúde Unit. Outubro; 6(2), 13-22.

Camacho, A. C. L. F.; et al., (2020). A tutoria na educação à distância em tempos de COVID-19: orientações relevantes. Research, Society and Development, [S. l.], 9(5), e30953151, DOI: 10.33448/rsd-v9i5.3151.

Carvalho G A O, Sousa G P, Pierote J J A, Caetano V S, Lima D E O, Costa I V S, Silva F A J C, \& Lima LF C. (2020). Anxiety as an ethological factor of bruxism - literature. RSD [Internet]. Apr; 9(7), e95973925.

Fernandes, S. M et al., (2020). O ensino a distância no Brasil: alguns apontamentos. Research, Society and Development, [S. l.], 9(1). e21911551, DOI: $10.33448 /$ rsd-v9i1.1551.

Kina M, Vilas Boas T P, Tomo S, Fabre A F, Simonato LE, Boer N P, et al. Lesões cervicais não cariosas: protocolo clínico. Arch Health Invest. 4(4): 21-28.

Maltarollo T H, Pedron I G, Medeiros J M F, Kubo H, Martins J L, \& Shitsuka C. (2020). The dental erosion is a problem!. RSD [Internet]. Mar; 9(3), e168932723.

Marinescu I R, Popescu S M, Răghici E C, Scrieciu M, Mercuț V, Turcu A A, \& Nicola A G. (2017). Etiological Aspects of Noncarious Dental Lesions. Curr Health Sci J. Jan-Mar;43(1):54-61.

Nascimento M M, Dilbone D A, Pereira P N, Duarte W R, Geraldeli S, \& Delgado A J. (2016). Abfraction lesions: etiology, diagnosis, and treatment options. Clin Cosmet Investig Dent. May 3;8:79-87.

Oliveira J P S. (2020). Os principais fatores de risco associados a lesões cervicais não cariosas. Escola Bahiana de Medicina e Saúde Pública. 
Peumans M, Politano G, \& Meerbeek B V. (2020). Treatment of noncarious cervical lesions: when, why, and how. The International Journal of Esthetic Dentistry. 15(1), 16.

Pinheiro J C, Silva L A M, Silva G G, Gonçalves G C, Leite R B, \& Almeida D R M F. (2020). Conceitos sobre o diagnóstico e tratamento das lesões cervicais não cariosas: revisão de literatura. Revista Pró-UniverSUS. Jan./Jun; 11 (1): 103-108.

Rocha, S. S. D. et al., (2020). A Educação a Distância na era digital: tipologia, variações, uso e possibilidades da educação online. Research, Society and Development, [S. l.], 9(6). e10963390, . DOI: 10.33448/rsd-v9i6.3390.

Santo, E. E. (2016) Ensinar e aprender na Educação a Distância: um estudo exploratório na perspectiva das práticas tutoriais. Research, Society and Development, [S. l.], 3(2), 92-114, . DOI: 10.17648/rsd-v3i2.16.

Shaalan O O, Abou-Auf E, \& El Zoghby A F. (2017). Clinical evaluation of flowable resin composite versus conventional resin composite in carious and noncarious lesions: Systematic review and meta-analysis. J Conserv Dent. Nov-Dec;20(6):380-385.

Shitsuka C, Mendes F M, Corrêa M S, \& Leite M F. (2015). Exploring some aspects associated with dentine hypersensitivity in children. ScientificWorldJournal. ;2015:764905. doi: 10.1155/2015/764905.

Shitsuka C, Palma L F, Pedron I G, Polotow T G G, Barros M P, Leite M F, \& CorrÊa M S N P. (2020). Salivary profile of children with erosive tooth wear: a transversal study. Braz Oral Res. Sep 4;34:e115. doi: 10.1590/1807-3107bor-2020.vol34.0115.

Shitsuka C, Tello G, \& Corrêa. M S N P. (2016). Desgaste dentario erosivo en bebés, niños y adolescentes: una visión contemporánea. Odontología,

Silva L L C, Pereira E K G, Silva D F, Rodrigues R F, Hora S L, \& Lins F C R. (2020). Restorative treatment for non-carious cervical lesions - part 2. RSD [Internet]. Nov; 9(11), e55991110236.

Warreth A, Abuhijleh E, Almaghribi M A, Mahwal G, \& Ashawish A. (2020). Tooth surface loss: A review of literature. Saudi Dent J. Feb; 32(2):53-60.

Xavier A F C, Pinto T C A, \& Cavalcanti A L. (2012). Lesões Cervicais não cariosas: um panorama atual. Rev. Odontol. Univ. 24(1): 57-66. 